\title{
Serum Levels of MicroRNA371a-3p: A Highly Sensitive Tool for Diagnosing and Staging Testicular Germ Cell Tumours: A Clinical Case Series
}

\author{
Petra Anheuser $^{a} \quad$ Arlo Radtke $^{c} \quad$ Christian Wülfing $^{b} \quad$ Jennifer Kranz ${ }^{d}$ \\ Gazanfer Belge ${ }^{c}$ Klaus-Peter Dieckmann ${ }^{a, b}$ \\ ${ }^{a}$ Klinik für Urologie, Albertinen-Krankenhaus, and ${ }^{b}$ Klinik für Urologie, Asklepios Klinik Altona, Hamburg, \\ ${ }^{c}$ Fachbereich Biologie, Universität Bremen, Bremen, and d Klinik für Urologie und Kinderurologie, St. Antonius \\ Hospital, Eschweiler, Germany
}

\section{Keywords}

Testicular germ cell tumour - MicroRNA371a-3p .

Biomarker · Treatment monitoring

\begin{abstract}
Introduction: MicroRNA (miR)371a-3p was suggested to be a sensitive and specific new serum biomarker of germ cell tumours (GCTs); however, its clinical usefulness remains unproven. Patients, Methods: In 312 consecutive cases with various testicular diseases, serum levels of miR371a-3p were measured. Measurement results became available only after completion of treatment. Five patients with testicular seminoma were selected for review because of unanticipated clinical courses. Results: In each two patients, elevated miR levels heralded undetected primary testicular GCT and metastases despite inconclusive radiological findings. In one case, a normal miR371a-3p level correctly pointed to the absence of metastases contrary to clinical assessment. In all cases, knowledge about the miR371a-3p levels would have altered the clinical management. Conclusions: These cases highlight the exceptional usefulness of the new GCT biomarker. In contrast to classical markers, miR371a-3p can identify primary testicular GCT. The marker can aid in clinical decision making in cases with ambiguous clinical findings. @ 2017 The Author(s)

Published by S. Karger AG, Basel
\end{abstract}

\begin{tabular}{ll}
\hline KARGER & $\begin{array}{l}\text { ( ) 2017 The Author(s) } \\
\text { Published by S. Karger AG, Basel }\end{array}$ \\
$\begin{array}{l}\text { E-Mail karger@karger.com } \\
\text { www.karger.com/uin }\end{array}$ & $\begin{array}{l}\text { Karger } \\
\text { This article is licensed under the Creative Commons Attribution- } \\
\text { NonCommercial-NoDerivatives 4.0 International License (CC BY- } \\
\text { NC-ND) (http://www.karger.com/Services/OpenAccessLicense). } \\
\text { Usage and distribution for commercial purposes as well as any dis- } \\
\text { tribution of modified material requires written permission. }\end{array}$
\end{tabular}

\section{Introduction}

The primary diagnosis of testicular germ cell tumours (GCTs), as well as the staging of the disease, is based on 3 examination techniques: clinical examination, imaging studies and measuring serum levels of the biomarkers beta human chorionic gonadotropin, alphafetoprotein and lactate dehydrogenase [1]. The accurate assessment of the disease is crucial for selecting the appropriate treatment and thus forms the basis for cure. As a matter of fact, all of the 3 examination techniques listed above are hampered by methodological problems. Traditional clinical examination is the least sensitive method because it is highly dependent on personal experience. Medical imaging, for example, CT and MRI can accurately document metastases larger than $1 \mathrm{~cm}$ in diameter but significantly lack sensitivity in diagnosing small secondaries [2,3]. Moreover, the specificity of medical imaging is unfavourable, too, because $10-20 \%$ false-positive results may occur $[4,5]$. Serum tumour markers are expressed only by $50 \%$ of the GCT patients and certain subgroups, for example, seminoma and teratoma do not express alpha-fetoprotein $[6,7]$. Lactate dehydrogenase is largely unspecific with a high number of false positive findings and is therefore recommended

Prof. Dr. med. Klaus-Peter Dieckmann

Klinik für Urologie

Asklepios Klinik Altona, Paul Ehrlich Strasse 1

D-22763 Hamburg (Germany)

E-Mail DieckmannKP@t-online.de 

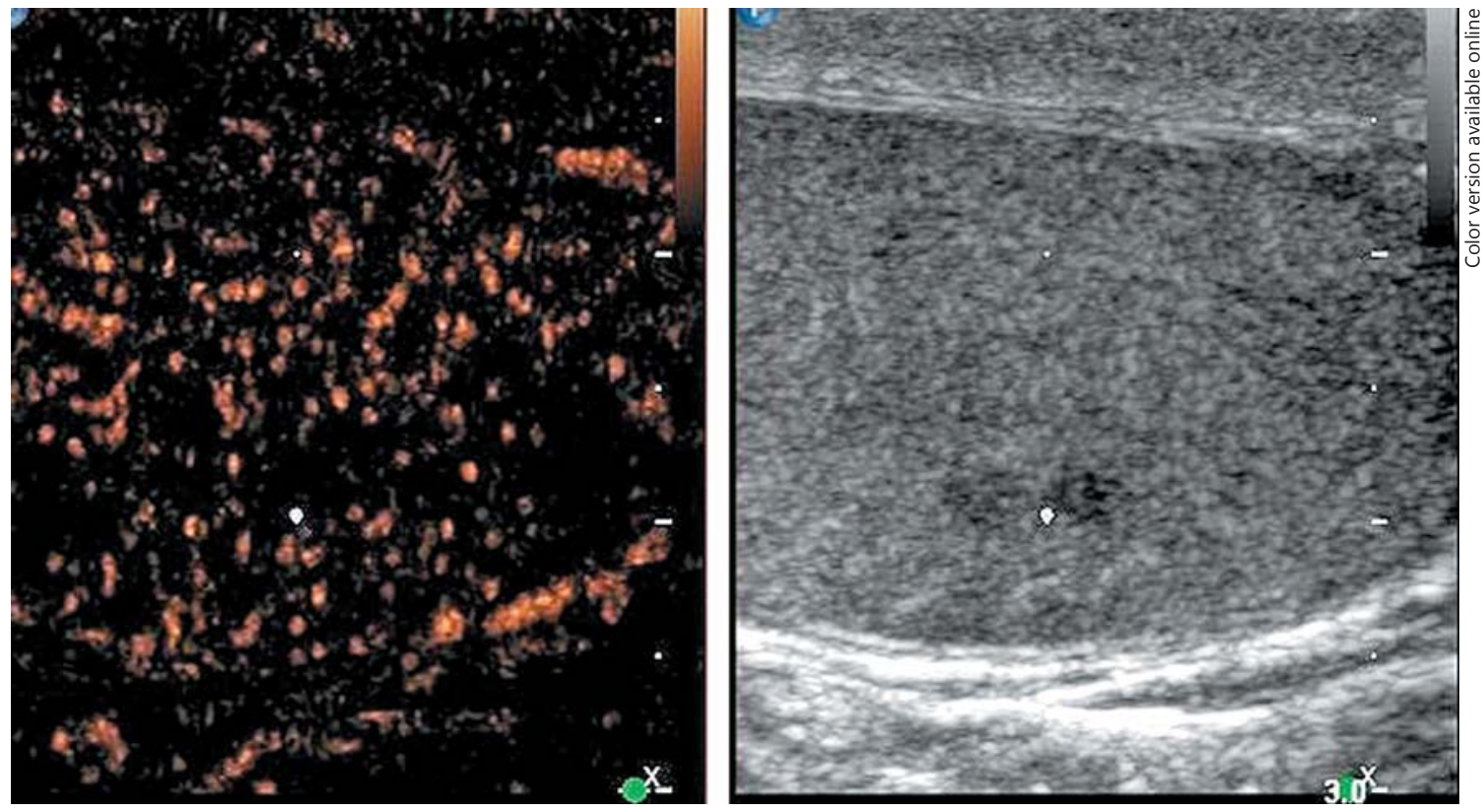

Fig. 1. B-mode (right side of figure) and contrast-enhanced ultrasonographic (CEUS, left side) images of left testis of patient \#1. The white dot denotes a small hypo-echoic lesion suspicious of neoplasia in the B-mode scan. However, CEUS does not reveal any enhancement of air bubbles in the corresponding region, and thus the presence of a testicular tumour was excluded. only for selected subgroups of GCT [6]. In 2011, microRNAs (miRs)371-3 and 302/367 were suggested as new serum biomarkers of GCT [8] and subsequent investigations revealed an extraordinary sensitivity and specificity of miR371a-3p, thereby rendering this marker as a very promising novel tool for diagnosing and staging testicular GCTs [9-11]. While investigating this marker systematically in a larger patient cohort, we recently experienced a number of isolated cases where the new marker miR371a-3p proved extremely helpful, clinically. We document these cases to demonstrate the exceptional usefulness of the new biomarker.

\section{Methods, Patients}

From January 2013 through December 2016, a total of 312 patients with scrotal abnormalities underwent serum examination within an ongoing study on the usefulness of miR371a-3p as a biomarker of testicular cancer. Of the patients, 163 had testicular GCTs, and 149 had various benign scrotal diseases with epididymitis, hydrocele and spermatocele representing the most frequent diagnoses. Information about one quarter of both, the GCT patients and those with benign diseases, has been included in previous communications $[12,13]$. Results of the measurements of miR371a-3p of the other patients will be reported elsewhere. Here we separately document 5 individual patients with testicular seminoma, aged 23-43 years, who were selected for this report because of their unanticipated clinical courses. We retrospectively correlated the miR-levels with the individual clinical features and we wondered if knowledge about the marker levels would have influenced therapeutic decisions differently.

The quantitative PCR technique employed in all of the patients has been detailed earlier [14]. Serum levels of a relative quantification (RQ) value $<5$ were defined as normal range [13]. The laboratory technique is still in its phase of clinical evaluation. So, all of the serum samples were stored deep-frozen at $-80^{\circ} \mathrm{C}$ until processing. Accordingly, in each of the 5 cases reported here, the results of miR measurements became available only after completion of treatment, and thus, the serum levels reported did not have any bearing on clinical decision-making.

\section{Results}

In all of the 5 cases, knowledge about the miR371a-3p serum levels would have significantly impacted the clinical management. Clinical details are given in following case descriptions.

1st patient: this 43-year-old man presented with leftsided testicular discomfort. Contrast-enhanced scrotal ultrasonography demonstrated unspecific irregularities of the intratesticular echo pattern (Fig. 1). Scrotal MRI was likewise inconclusive. The classical serum tumour markers were all in the normal range. Therefore, the patient was put on a surveillance schedule. As the patient 

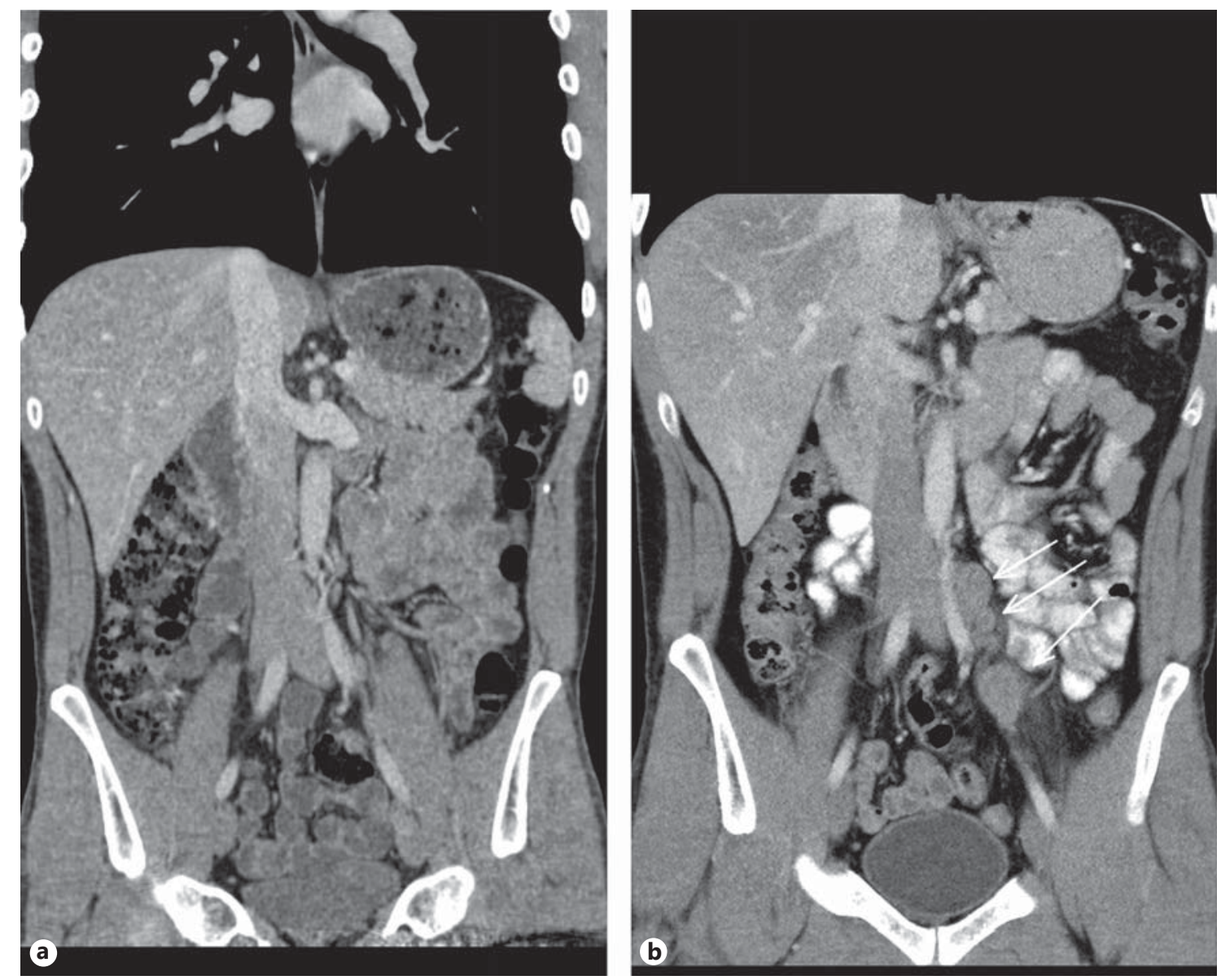

Fig. 2. a Patient \#3, left-sided testicular seminoma. Abdominal CT reveals unspecific small lymph node lateral to left iliac artery. Radiological diagnosis: no metastases detected. b Same patient, ab- dominal CT 26 months after adjuvant Carboplatin treatment: a chain of enlarged lymph nodes lateral to left iliac artery (arrows). Diagnosis: retroperitoneal relapse of seminoma. moved to another city, he was lost to follow-up. But, enquiries disclosed that orchiectomy for classical seminoma (pT1, clinical stage [CS] 1) was performed on him at another institution 4 months after our primary assessment. The serum level of miR371a-3p at the time of primary examination was RQ $=39.45$ which is much higher than the normal range. Knowledge about the elevated miR-level would clearly have changed decision-making. No follow-up measurements of miR371a-p were available.

2nd patient: this 36-year-old male, was enrolled as a healthy control in our previous study [13]. He had a serum level of $\mathrm{RQ}=21.45$ at the time of blood examination. Clinically, he was in a healthy condition with no signs of any testicular disease. To our surprise, he presented with a full-blown testicular seminoma (pT1, CS1) only 1 year later. At that time, the $\mathrm{miR}$ level had risen to $\mathrm{RQ}=963.9$ to drop to $R Q=0.01$, postoperatively. Further management consisted of surveillance and after 1 year of followup, the patient is well and the serum level of miR371a-3p remained low at $\mathrm{RQ}=0.0$.

3rd patient: this 31-year-old patient underwent orchiectomy for testicular seminoma (pT3, CS1). Although markers were negative and no metastases were detected radiologically (Fig. 2a), the patient received adjuvant single-shot carboplatin therapy because of spermatic cord invasion of the primary and on grounds of personal choice of the patient. Twenty-six months thereafter he presented with a bulky retroperitoneal relapse (Fig. $2 b$ ). He was salvaged with 3 courses of BEP (bleomycin, etoposide and cisplatin) chemotherapy. It is to be noted here that the miR371a-3p level was at RQ $=3081.8$ before orchiectomy and it continued to remain elevated at RQ = 351.8 five days after orchiectomy. 
Then, no further decrease was recorded. At the time of the clinically apparent relapse, the level had risen to $\mathrm{RQ}=4240.3$ and it decreased to normal levels at the completion of salvage therapy and remained stable during the later course. This case was briefly reported previously [13].

4th patient: this 37-year-old patient had left-sided orchiectomy for a presumed occult germ cell tumour. Histologically, only germ cell neoplasia in situ (TNM: pTis) was found in the specimen. CT revealed a $1.8 \mathrm{~cm}$ lymphadenopathy in the para-aortal template. All of the classical serum tumour markers were negative. As histopathologically no invasive GCT was found in the left orchiectomy specimen, the lymphadenopathy was considered unspecific corresponding to a CS1. Accordingly, the patient was put on a surveillance schedule. Four months later, the lymphadenopathy had slightly increased (Fig. 3). Therefore, a laparoscopic lymphadenectomy was performed. Histologically, the specimen revealed a pure seminomatous metastasis. The patient received 3 cycles of BEP chemotherapy subsequently. Before orchiectomy, the miR371a-3p serum level was RQ $=74.4$ and it was found to decrease to $R Q=20.4$ three days postoperatively, thus clearly lying in the supra-normal range. Prior to lymphadenectomy, the level had increased to $R Q=58.5$ and it dropped to $\mathrm{RQ}=0.0$ after surgical excision of the node to remain there during the later course.

5th patient: this 23-year-old man underwent orchiectomy for marker-negative testicular seminoma at another institution. CT revealed intrathoracic nodules suspicious for mediastinal lymph node metastases. Accordingly, 4 cycles of BEP chemotherapy were administered. Restaging revealed unchanged mediastinal lymphadenopathy (Fig. 4). Then, the patient was referred to our institution for further management. Thorough pulmonological examination including transbronchial biopsy disclosed sarcoidosis-like lymphadenopathy, and thus metastatic disease was excluded. In view of these results, it must retrospectively be assumed that this patient had sarcoidosis synchronously with seminoma rather than having metastatic disease. Accordingly, the serum miR371a3 p level was $R Q=0.0$ at the time of re-staging after chemotherapy. Unfortunately, no miR-levels were measured at the time of first presentation. Although speculative, one would assume that miR371a-3p levels had probably been low after orchiectomy for seminoma because the mediastinal lymphadenopathy had not changed ever since. By retrospective consideration, the normal marker level would have indicated the absence of metastases at the outset and accordingly, the patient could have been



Fig. 3. Abdominal CT of patient \#4, 4 months after orchiectomy: para-aortal lymphadenopathy. Laparoscopic excision revealed seminoma.

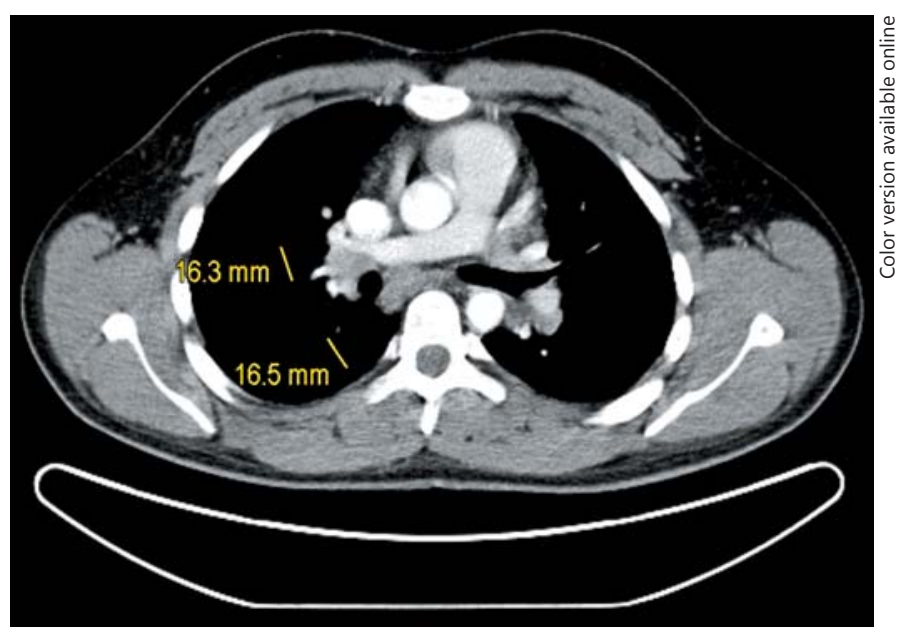

Fig. 4. Chest CT of patient \#5 after 4 cycles of cisplatin-based chemotherapy: hilar lymphadenopathy. Transbronchial biopsy revealed sarcoidosis. 
spared unwarranted chemotherapy and excessive diagnostic procedures apart from emotional distress from extended treatment.

\section{Discussion}

The central result of this report is the documentation of the exceptional usefulness of the new serum biomarker miR371a-3p in diagnosing and staging testicular GCT. In 4 of the 5 cases, the marker had indicated active germ cell cancer despite negative or inconclusive findings with the classical staging methods. In all of the 4 cases, the serum levels were clearly above the cut-off level of RQ $<5$ [13]. It is worthy to note that 2 cases (\#1-2) represented missed primary diagnoses of testicular GCT. In the first case, unspecific clinical symptoms along with inconclusive scrotal imaging precluded the timely diagnosis of testicular GCT. Given the elevated miR-level, surgery could have been performed earlier. Strikingly, the second case had an elevated miR level more than 1 year before the clinical manifestation of the malignancy. As clinically no testicular anomalies were detectable at the time of blood examination, the elevated serum level of miR371a-3p had obviously been preceding the invasive cancer for that long time. In cases \#3 and \#4, small retroperitoneal metastases were missed out with the current radiological methods, while the elevated miR371a-3p levels had already pointed to the presence of metastatic disease. It thus appears that elevated miR371a-3p levels are highly sensitive and are capable of detecting GCT (primaries and secondaries) much earlier than radiological methods can.

Conversely, serum levels of miR371a-3p can also help to exclude metastatic GCT as documented in case \#5, where the normal level of miR371a-p could have helped to correctly assess ambiguous radiological findings and thus exclude metastatic disease.

In conclusion, these cases clearly highlight the considerable clinical value of measuring serum levels of miR371a-3p in GCTs $[15,16]$.
We clearly acknowledge shortcomings of our retrospective documentation mainly lacking information regarding clinical details of the patients and particularly missing repeat measurements of miR371a-3p for comparison with the index miR-level. So, part of our conclusions is built only on supposition. Notwithstanding, there is accumulating evidence that even small amounts of GCT tissue could cause serum elevation of miR371a$3 p$, highlighting the extraordinary sensitivity of this marker [12]. Clinically, it appears that the new marker may not only aid in revealing metastatic disease but other than the classical markers [17] - it may also aid in assessing primary testicular masses. It is to be noted here that the marker elevation appears to precede the clinical manifestation of primary GCT by many months. Measuring serum levels of miR371a-3p apparently represents a useful tool for timely uncovering ambiguous clinical presentations of testicular cancer disease [18], and this novel biomarker clearly deserves further clinical investigation.

\section{Acknowledgement}

Dr. Raphael Ikogho helped in compiling the clinical case histories. Dirk Mumm and the Laboratory staff of Albertinen-Zentrallabor provided valuable assistance in processing and storing the serum samples.

\section{Disclosure Statement}

G. B. and K.-P.D. possess shares of miRdetect GmbH, a Bremen-based biotech company aiming to develop a commercially available laboratory test for measuring miR levels in body fluids. No other author has declared potential conflicts of interests.

\section{Funding Sources}

This study was supported by Wilhelm Sander Stiftung, Munich, and Albertinen-Stiftung, Hamburg.

\section{References}

1 Albers P, Albrecht W, Algaba F, Bokemeyer C, Cohn-Cedermark G, Fizazi K, Horwich A, Laguna MP, Nicolai N, Oldenburg J: Guidelines on testicular cancer: 2015 update. Eur Urol 2015;68:1054-1068.

2 Hilton S: Contemporary radiological imaging of testicular cancer. BJU Int 2009;104(9 pt B): $1339-1345$.
3 Heidenreich A, Albers P, Classen J, Graefen M, Gschwend J, Kotzerke J, Krege S, Lehmann J, Rohde D, Schmidberger H, Uder M, Zeeb H; Association of Urologic Oncology of the German Cancer Society: imaging studies in metastatic urogenital cancer patients undergoing systemic therapy: recommendations of a multidisciplinary consensus meeting of the asso- ciation of urological oncology of the German cancer society. Urol Int 2010;85:1-10.

4 Donohue JP, Thornhill JA, Foster RS, Bihrle R, Rowland RG, Einhorn LH: The role of retroperitoneal lymphadenectomy in clinical stage B testis cancer: the Indiana university experience (1965 to 1989). J Urol 1995;153: 85-89. 
5 Secil M, Altay C, Basara I: State of the art in germ cell tumor imaging. Urol Oncol 2015; 34:156-164.

6 Gilligan TD, Seidenfeld J, Basch EM, Einhorn LH, Fancher T, Smith DC, Stephenson AJ, Vaughn DJ, Cosby R, Hayes DF: American society of clinical oncology clinical practice guideline on uses of serum tumor markers in adult males with germ cell tumors. J Clin Oncol 2010;28:3388-3404.

7 Neumann A, Keller T, Jocham D, Doehn C: [Human placental alkaline phosphatase (HPLAP) is the most frequently elevated serum marker in testicular cancer]. Aktuelle Urol 2011;42:311-315.

8 Murray MJ, Halsall DJ, Hook CE, Williams DM, Nicholson JC, Coleman N: Identification of microRNAs from the miR-371 373 and mir-302 clusters as potential serum biomarkers of malignant germ cell tumors. Am J Clin Pathol 2011;135:119-125.

9 Belge G, Dieckmann KP, Spiekermann M, Balks T, Bullerdiek J: Serum levels of micro-
RNAs mir-371-3: a novel class of serum biomarkers for testicular germ cell tumors? Eur Urol 2012;61:1068-1069.

10 Murray MJ, Huddart RA, Coleman N: The present and future of serum diagnostic tests for testicular germ cell tumours. Nat Rev Urol 2016;3:715-725.

11 van Agthoven T, Looijenga LH: Accurate primary germ cell cancer diagnosis using serum based microRNA detection (ampTSmiR test). Oncotarget 2016, Epub ahead of print.

12 Dieckmann KP, Spiekermann M, Balks T, Ikogho R, Anheuser P, Wosniok W, Loening T, Bullerdiek J, Belge G: MicroRNA miR-371a$3 p$ - a novel serum biomarker of testicular germ cell tumors: evidence for specificity from measurements in testicular vein blood and in neoplastic hydrocele fluid. Urol int 2016;97:76-83.

13 Dieckmann KP, Radtke A, Spiekermann M, Balks T, Matthies C, Becker P, Ruf C, Oing C, Oechsle K, Bokemeyer C, Hammel J, Melchior S, Wosniok W, Belge G: Serum levels of
microRNA miR-371a-3p: a sensitive and specific new biomarker for germ cell tumours. Eur Urol 2017;71:213-220.

14 Spiekermann M, Dieckmann KP, Balks T, Bullerdiek J, Belge G: Is relative quantification dispensable for the measurement of $\mathrm{mi}$ croRNAs as serum biomarkers in germ cell tumors? Anticancer Res 2015;35:117-121.

15 Bezan A, Gerger A, Pichler M: MicroRNAs in testicular cancer: implications for pathogenesis, diagnosis, prognosis and therapy. Anticancer Res 2014;34:2709-2713.

16 Ellinger J, Müller SC, Dietrich D: Epigenetic biomarkers in the blood of patients with urological malignancies. Expert Rev Mol Diagn 2015; 15:505-516.

17 Mir MC, Pavan N, Gonzalgo ML: Current clinical applications of testicular cancer biomarkers. Urol Clin North Am 2016;43:119-125.

18 Ling H, Krassnig L, Bullock MD, Pichler M: MicroRNAs in testicular cancer diagnosis and prognosis. Urol Clin North Am 2016;43:127134

Serum Levels of miR371a-3p 